Review

\title{
Peripheral nerve injury treatments and advances: One Health perspective
}

\author{
Bruna Lopes 1,2, Patrícia Sousa 1,2, Rui Alvites 1,2, Mariana Branquinho 1,2, Ana Sousa 1,2, Carla Mendonça 1,2, Luís \\ Miguel Atayde 1,2, Ana Lúcia Luís 1,2, Artur S.P. Varejão ${ }^{3,4}$, and Ana Colette Maurício ${ }^{1,2 *}$
}

${ }^{1}$ Centro de Estudos de Ciência Animal (CECA), Instituto de Ciências, Tecnologias e Agroambiente (ICETA) da Universidade do Porto, Praça Gomes Teixeira, Apartado 55142, 4051-401 Porto, Portugal; brunisabe195@gmail.com (B.L.); pfrfs_10@hotmail.com (P.S.); ruialvites@hotmail.com (R.A.); m.esteves.vieira@gmail.com (M.B.); anacatarinasoaressousa@hotmail.com (A.S.); cmmendonca@icbas.up.pt (C.M.); ataydelm@gmail.com (L.M.A.)

2 Departamento de Clínicas Veterinárias, Instituto de Ciências Biomédicas de Abel Salazar (ICBAS), Universidade do Porto (UP), Rua de Jorge Viterbo Ferreira, no 228, 4050-313 Porto, Portugal.

3 Department of Veterinary Sciences, University of Trás-os-Montes e Alto Douro, UTAD, Quinta de Prados,5000-801 Vila Real, Portugal.

${ }^{4}$ CECAV, Centre for Animal Sciences and Veterinary Studies, University of Trás-os-Montes e Alto Douro, Quinta de Prados, 5000-801 Vila Real, Portugal.

* Correspondence: acmauricio@icbas.up.pt; Tel.: +351-91-9071286.

\begin{abstract}
Peripheral nerve injuries (PNI) can have several etiologies, such as trauma and iatrogenic interventions that can lead to the loss of structure and/or function impairment. These changes can cause a partial or complete loss of motor and sensory functions, physical disability, and neuropathic pain, what in turn can affect the quality of life. For those reasons, PNI is a major public health concern. This review aims to revisit the concepts associated with the PNI. First, the anatomy of the peripheral nerve is detailed to explain the different types of injury. Then, some of the available therapeutic strategies are explained, including surgical methods, pharmacological therapies, and the use of cell-based therapies alone or in combination with biomaterials in the form of tube guides. Nevertheless, even with the various available treatments, it is difficult to achieve a perfect outcome with complete functional recovery. This review aims to explain the urge for new approaches and to understand the methods to evaluate nerve regeneration in a One Health perspective. In vitro models followed by in vivo models are very important to be able to translate the achievements to human medicine.
\end{abstract}

Keywords: Mesenchymal stem cells; Nerve Guide Conduits; Nerve recovery; One Health; Peripheral nerve injury; Secretome.

\section{Introduction}

The coordination and communication throughout the body mainly depends on the highly specialized nervous system, which gives the possibility to respond to different environmental changes [1]. This appropriate response is generated when the central nervous system (CNS) obtains information from the receptors sent via afferent neurons. Then, the CNS processes the information and the efferent neurons send signals to the different effector organs of the body [1]. The nervous system is divided in two major regions: CNS and peripheral nervous system (PNS) [2]. The brain and spinal cord constitute the CNS, which is responsible for centrally process the information arriving from the periphery [1]. The PNS is the extensive grid of nerves that functionally integrate diverse parts of the body with the CNS [1,2]. The PNS results from the combination of the ventral root and the dorsal root both originated from the spinal cord. The first includes 
the motor neurons and the later the sensory neurons, which cell bodies reside in the dorsal root ganglion. In the ventral horn of the spinal cord and in specific nuclei of the brainstem are located the cell bodies of the motor neurons. The sensory and motor neurons axons can communicate with distant target organs. In addition, the PNS is more easily regenerated in comparison to the CNS [3] due to their distinct functional environments, integrity of the injured nerve, age of the affected subject and type of injury sustained. PNI is a neurological condition that seriously threatens both human and animal patients, leading to serious and long-term functional and physiological disabilities [4, 5]. Neuropathic pain is one of the most complicated pain syndromes to regulate and can cause this kind of disorder can cause devastating impacts on patients' daily routines and impair their emotional well-being $[6,7]$.

Peripheral nerve fibers are very delicate structures, making them easily damaged by different types of compressions [2]. The consequent communicational abnormalities between the brain and the target muscles/organs are the manifestation of the damage [2]. The nerve defect can have multiple origins, such as iatrogenic (medical or surgical) and traumatic $[5,8]$. A big percentage of PNI is associated to poor functional outcomes, insufficient nerve recovery, and the loss of sensory and motor function. These are followed by partial recovery, muscle atrophy, chronic pain, and profound weakness [9]. Often, axons have to regenerate over long distances at a slow rate of 1-3 mm per day to reinnervate and reach distal motor endplates [10]. For that reason, the regenerative process takes time, especially without any external intervention [4, 11]. Also, there are additional factors that impair nerve regeneration after PNI, such as the loss of extensive amounts of nerve tissue and prolonged denervation of the proximal nerves that enhance the probability of irreversible atrophy of the innervated organs [4].

The types of nerve injuries are classified based on the severity and extent of the damage, creating different outcome possibilities and the recovery depends on the severity $[5,12]$. To promote better outcomes, it is important to guarantee a rapid intervention. However, even doing so, the prolonged denervation of the nerve segments can lead to little recovery rates and to other disabilities [5, 12].

Treatments are mainly microsurgical interventions either with direct repair, tension free end-to-end suture, and the gold standard technique, the use of autologous nerve grafts to repair larger gaps $[8,13,14]$. The use of the latter reduces the risk of immunological rejection and provides a native tissue microenvironment that triggers a positive therapeutic effect $[4,9,11]$. However, it has some limitations, such as donor site morbidity, the lack of donor sources, sensory loss, scarring and neuroma formation [4, 9, 11]. Recently, many studies focused on establishing new methods to promote axonal regeneration, ideally without sacrificing other healthy functioning nerves, and improving the PNI outcome. Nerve guide conduits (NGCs) are a viable alternative to autologous nerve grafting. These have many advantages, in comparison with autologous nerve graft, such as unrestricted source of supplement, easy fabrication, repeatability and prevention of donor site morbidity [11]. The use of Schwann cells (SCs) or mesenchymal stem cells (MSCs) in synthetic permeable nerve conduits is one of the focuses for an ideal treatment [4]. The animal model has the advantage of having multimodal approaches and different combinations of methods to study axonal regeneration. In addition, in the One Health context, assessment of nerve regeneration using animals provides information to translational research and future therapeutic options available for humans [15].

The aim of this review is to address the PNI and focus on the treatments and advances achieved in peripheral nerve regeneration, especially using mesenchymal stem cells and biomaterials. Besides, the authors want to discuss the use of MSCs' secretome as other possible therapy, as well as, advantages and disadvantages from the conventional methods. Another approach is the One Health topic, since most of the studies use animals. 
Therefore, it is important to discuss the relevance of using animals to achieve an optimized treatment, for both veterinary and human medicine. In addition, in vivo models provide more data about nerve regeneration using behavior and functional assessments. Since PNI can be chronic and lead to serious problems in the quality of life, other recent studies will be mentioned, where the dietary and different types of exercise can help reaching better outcomes.

\section{Peripheral nerve anatomy}

Each peripheral nerve is constituted by multiple longitudinal arrangements of axons, named as fascicles, and covered by three layers of connective tissue. These layers support the fascicles and contain blood vessels that provide trophic support for the nerve fibers [1, 5 , 9]. The epinerium is the outermost layer, which comprises all fascicles that form the peripheral nerve [9]. Areolar connective tissue, that allows the nerve contraction and expansion, is the main constituent of the epinerium [9]. In addition, depending on the size and the specific type of nerve, it represents between $30-70 \%$ of the nerve trunk sectional area [5]. All fascicles are coated by the inner portion of the epinerium, which contains blood vessels that irrigate the nerve and some adipose tissue [5]. The mechanical protection and anatomical shape are provided by the external layer that coats the entire nerve [5].

The middle layer is the perinerium, a thin and dense connective layer that envelops each fascicle individually. Therefore, helps to maintain homeostasis in the structure and to protect the endoneurial environment $[5,9]$. Lastly, the inner layer is the endonerium, a thin layer of collagen fibers that surround each axon within the fascicle [9]. It contains a thin network of micro vessels, capillaries and possesses great elasticity, but this layer assures little mechanic protection [5]. In comparison with the perineurium and epinerium, which are circumferentially disposed, the endonerium has a longitudinal orientation [5]. The nerve fascicle is a group of axons surrounded by endonerium and each fascicle is covered by perinerium [5].

SCs are intimately associated with each individual myelinated axon [9]. These glial cells are capable of producing laminin-rich sheets of myelin, since they form a fatty multilayered membrane (several layers of SC membranes associated with secreted proteins) that isolate the axon. Myelin is very important to enhance the neural electrical impulses propagation rate along the nerve fiber. When the nerve fiber is demyelinated, the impulse moves in wave motions. On the other hand, in myelinated fibers the conduction occurs trough a saltatory propagation. Other myelin function that helps to assure a faster impulse is the increment of the electric resistance along the cell membrane. Along the myelinated fiber there is a heterogenous distribution of sodium channels, these channels are in bigger density at the Ranvier nodes and at a minor density in the internodal region. The inner regions lose small quantities of sodium to the extracellular fluid, which helps to maintain a separation of electrical charges, in opposition to the Ranvier nodes where the sodium channels density is higher. When sodium is in the extracellular fluid, a minimum quantity is enough to open the sodium channels, the ion penetrates the axon causing the action potential [5]. The action potential travels fast along the myelinated axon by jumping between the nodes of Ranvier. For axons of the same size, myelination accelerates nerve conduction in 20-100-fold, in comparison with unmyelinated axons, which is the reason why, normally, unmyelinated axons are smaller [9]. Besides the role in the myelination process, SCs are the main extrinsic mediators of peripheral nerve regeneration [9]. Another important cell type present in the peripheral nerve environment are the pericytes, which are contractile cells associated with the endothelial lining of microvasculature that modulate blood flow and capillary dilatation [9]. These cells help to maintain homeostasis 
both in the brain-nerve barrier and the endoneurial microenvironment [9]. The following figure (Figure 1) illustrates a schematic of peripheral nerve anatomy.

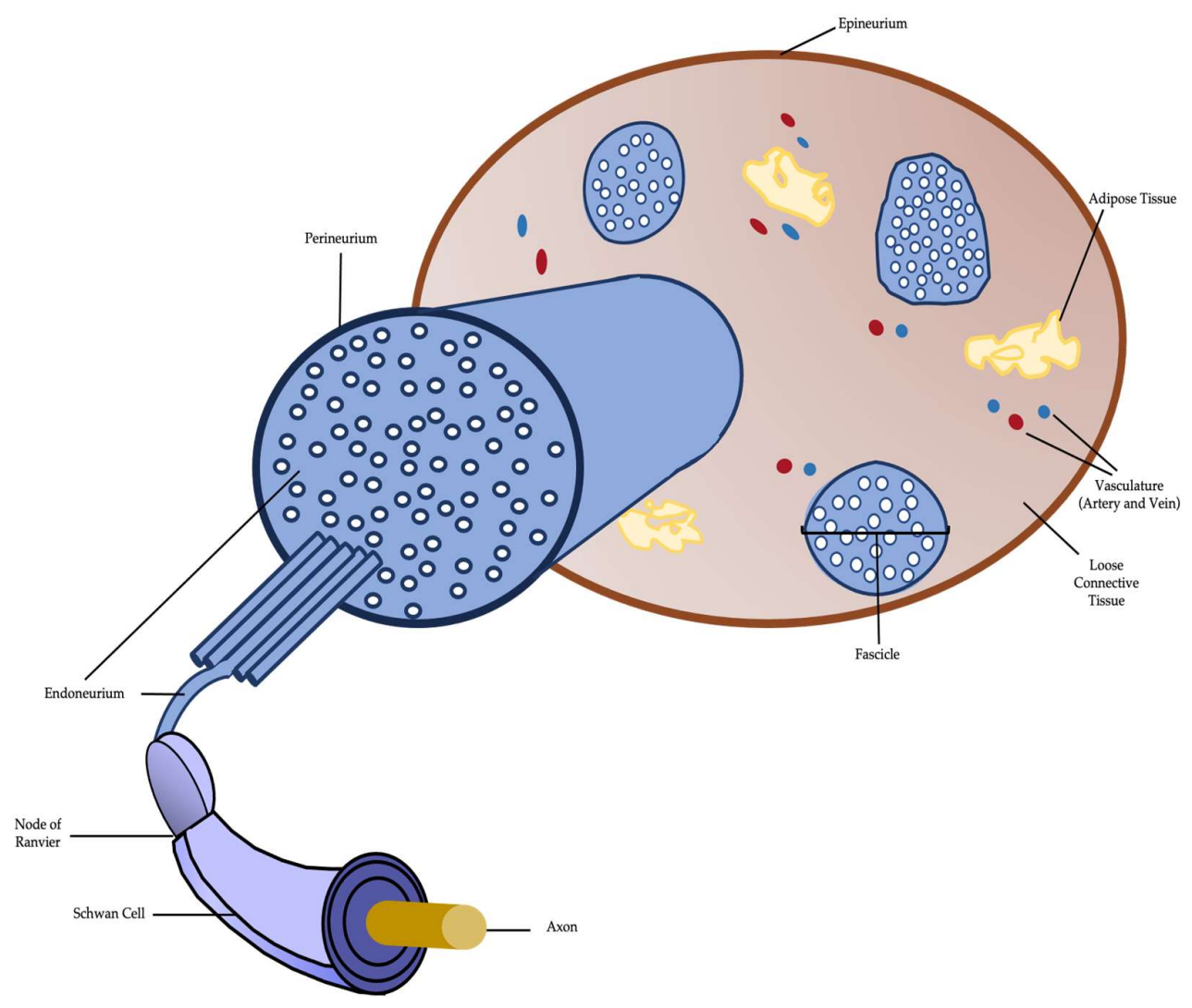

Figure 1: Schematic representation of a typical peripheral nerve.

\section{Type of lesion and scarring}

The first classification system was introduced by Sir Herbert Seddon, in 1943, which described three classes of PNI: neuropraxia, axonotmesis and neurotmesis, depending on the recovery time, prognosis and severity of nerve injury $[9,16,17]$. Class I, neuropraxia, is normally induced by ischemia or focal demyelination, due to traction or mild compression [9, 17]. In this situation, sensory and motor connection is lost, and the conduction of nerve impulses is blocked without any anatomical damage to the endoneurium, perineurium and epinerium. The recovery from this kind of injury is highly variable, but, normally a full recovery is achieved in days up to 3 months [16]. Class II or axonotmesis is caused by stretch, crush or percussion and is more severe than neuropraxia. This is characterized as a disruptive lesion both of the axon and its myelin coating and the perineurium and epineurium integrity remains [5]. Following, within 24-36 hours after PNI, Wallerian degeneration distally to the lesion site [16]. In this type of injury, sensory and motor deficits occur, and the nerve conductions fail, distally to the injury site. Although being a more severe lesion, the injured nerve stump maintains some integrity of the physiological structure and organization, and functional recovery is expected [16]. Class III or neurotmesis, is the most severe degree of PNI since it represents a nerve transection, where endonerium, perineurium and epineurium are totally disrupted. This lesion has a poor prognosis, since it leads to the rupture of the axon, myelin sheath and connective tissue $[16,17]$. In this case, recovery without surgery or other therapeutic option is almost non- 
existent because the disconnection of the two segments damages the collagen coatings and their guiding function implicating the normal regenerative sequence [5].

In 1951, Sunderland extended Seddon's classification from three to five degrees, based on increasing severity of damage in the nerve $[5,9]$. The first degree of PNI is the least severe and corresponds to neuropraxia from Seddon's scale [2, 16]. However, concerning axonotmesis, Sunderland divided this type of lesion in three different degrees: in the second-degree injury, the only structure that is disrupted is the axon, the endoneurial tubes, perineurium and epineurium remain intact $[2,16]$. In the third degree lesion, the axon is disrupted and the endoneurium's continuity is absent [16]. In the fourth degree, the epineurium is the only structure that maintains integrity, the axon, endoneurium and perineurium are injured. Finally, the fifth degree is the complete nerve defect as in neurotmesis, complete transaction of all layers [16].

The most recent update of PNI injuries was the introduction of a sixth-degree injury by Mackinnon and Dello, in 1988, (Figure 2) to the scheme of Sunderland [5]. This last degree corresponds to the occurrence of mixed injuries in the same nerve. There is the possibility to have lesions with different severities throughout the nerve's cross section and extension [5].

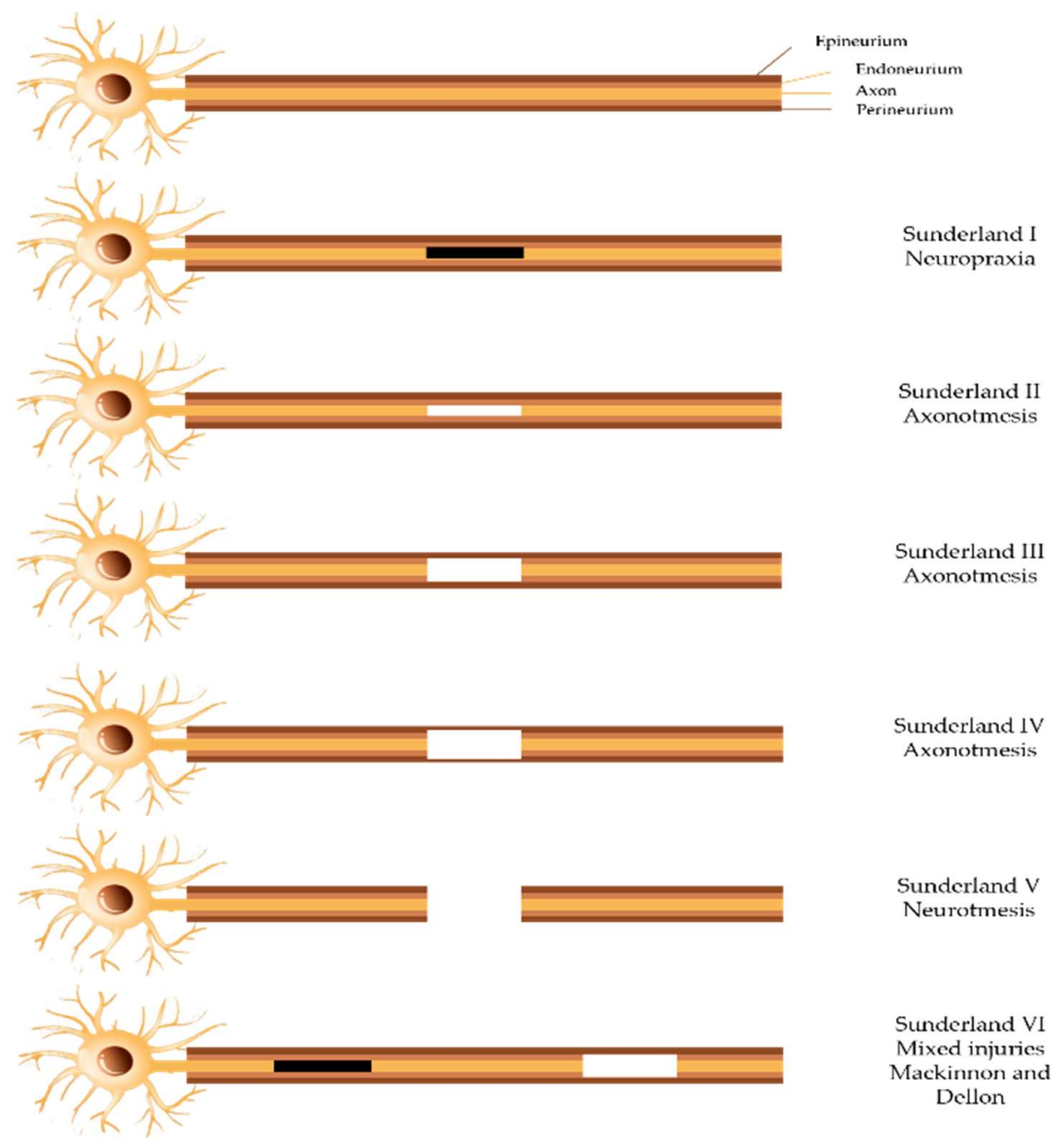

Figure 2: Schematic representation of the different injury grading systems for PNI.

\subsection{Events following peripheral nerve injury}


After the lesion, axonal outgrowth is coordinated by many factors, including the infiltration of immune cells, SCs phenotype transformation and neurovascular regeneration [4]. Macrophages, neutrophils, endothelial cells and SCs are very important in the different stages of the peripheral nerve repair mechanism. SCs proliferate, phagocyte debris in the lesion site and mobilize macrophages to promote the optimal regenerative surroundings [18, 19]. Also, SCs form the myelin sheath along peripheral nerves and are responsible for the formation of the bands of Bunger [20].

The axons from the distal portion start to degenerate because of a mechanism known as Wallerian Degeneration (WD), originally described by August Waller [21]. This degeneration begins with a flux of extracellular $\mathrm{Ca}^{2+}$ into the axon. For instance, the reduction of extracellular calcium or the ion $\mathrm{Ca}^{2+}$ channels blockage can attenuate the axon degeneration up to four days [19]. When increased, the intracellular calcium activates the calpains, proteases with the ability to cleave cytoskeletal proteins as the neurofilament. The discovery of a spontaneous mouse mutant, C57BL/WLDs, that exhibits very slow WD helped to understand this mechanism. WD is characterized by the breakdown and granular disintegration of the distal nerve stump after axon injury [19]. Upon an injury, the transport of nicotinamide mononucleotide adenyltransferase (NMNAT) by the cytoplasmic isoform, NMNAT2, from the cell body to axons to activate the nicotamide adenine dinucleotide (NAD+) is blocked. The short half-life of NMNAT2 explains the decrement of NAD+ levels that conducts to axonal disintegration [19]. WD results in the destruction of myelin and the enhance of SCs mitosis [22]. Following cytoskeleton and membrane degradation, the SCs surrounding the distal portion of the axon, shed their myelinated lipids [22]. Then, the debris from this degradation are removed by macrophages and SCs. Following axonal fragmentation and debris removal, the axons have to regrow throughout a bridge formed between the proximal and distal stumps in the injury site. This bridge is mainly constituted of extracellular matrix components and inflammatory cells that help axons to regrow towards their original targets and its length depends on the injury size. When the axons reach the bridge's edge, they initiate extending in the direction of the distal stump. Bands of Bunger are tubular structures present in the distal stump and important to guide the regrowing axons towards their original targets [20].

\section{Advances in the peripheral nerve treatment}

After a long regenerative period, full functional re-innervation without any complication is rare [2]. The process of regeneration, in severe injuries, is complicated and has limitations, which can result in the aggravation of muscular atrophy. For that reason, there is a need of surgery or other treatment, depending on the nerve gap and other factors [2]. The decision to perform a surgical intervention is complex: if the surgery is performed too soon, a lot of the potential for spontaneous recovery can be lost. However, confidence that the specific lesion has potential for a spontaneous recovery is not a risk-free decision since there is a period of time where the surgery can substantially improve recovery potential [5].

\subsection{Surgical treatment methods}

For PNI, the most common treatment method implies surgical approaches. For short gap PNI $(<1 \mathrm{~cm})$, neurorrhaphy is commonly used. This technique involves suturing the divided nerve, proximal and distal ends [1, 2, 12]. For that reason, it can only be used in 
short nerve gaps, since for a longer nerve gap this method would cause excessive tension leading to bad regenerative results. In the 1970s started the use of fibrin glue to mitigate the effects of the suture $[1,2,12]$. Comparing both techniques, fibrin glue provokes less granulomatous inflammation, decreased inflammatory response, quick application and the recovery is more a less the same. Nerve grafting is the most used technique for medium and large nerve gaps and recent studies showed that the reduction of stitches and the use of heterologous fibrin sealant can help minimize the trauma and, consequently, enhance nerve reconstruction $[23,24]$.

\subsubsection{Autograft: The current gold standard}

To bridge large nerve gaps $(>3 \mathrm{~cm})$, critical nerve injuries and more proximal injuries, the most common strategy is the autograft, which consists in the use of autograft taken from the patient's body from another nerve [2]. This technique implicates the use of functionally, but less crucial nerves, from various possible locations such as, sural, superficial cutaneous nerve or lateral femoral cutaneous nerve as a donor site [1, 2, 12, 25]. To choose the most suitable nerve to harvest, the size of the nerve gap, as well as the location of the proposed nerve repair and the donor-site morbidity have to be considered $[1,25]$.

The use of autologous nerve grafts functions as an immunogenically inert scaffold, which contributes to a stimulating and permissive environment, adhesion molecules and neurotrophic factors, that promote nerve regeneration [2]. This strategy has some limitations such as, donor site morbidity, need for an additional surgical procedure, loss of function from the donor site, scarring, possibility of painful neuroma and the narrow viability of the graft tissue $[1,2,12,25]$. In addition, this technique has other disadvantages such as limited supply, the need for a second incision to harvest the graft tissue and fascicle mismatch [1]. A recent study tested the autologous nerve transplantation with an aligned chitosan fiber hydrogel (ALG), filled with a bioactive peptide, RGI/KLT. RGI peptide is derived from BDNF and is important in motor neuron outgrowth. KLT acts as vascular endothelial growth factor (VEGF), important in the angiogenesis process [26]. The purpose was to improve a $15 \mathrm{~mm}$ sciatic nerve defect and the complex compound had positive functional recovery results. SCs were inoculated into different groups and after 48h, the SCs present in the ALG - RGI/KLT were orientated, in contrast with the cells from the control group that were disordered. The preliminary results prove that ALG helps to regulate the SCs directional growth. In addition, 12 weeks after the operation, the regenerated nerves were removed and stained. The number of regenerated nerve fibers was higher in the ALG - RGI/KLT group with greater diameter and thickness [23, 26]. Besides this work, other alternatives and more complex formulations are being studied for better regeneration of the peripheral nerve.

\subsubsection{Nerve Allograft}

When the nerve gap is bigger and graft length exceeds the accessible nerve autograft, other sources need to be taken into consideration. The use of a cadaveric or donor nerve, as an allograft, may be used as an alternative clinical option [2, 25]. Nerve allograft contributes with guidance and viable SCs that facilitate axons connection, beneficial to reinnervate the target tissue. Nonetheless, this technique has some disadvantages: it requires a period of 18-24 months (approximately) of immunosuppressive therapy, post implantation, in order to prevent graft rejection and to allow the host axons and SCs to regenerate along the allograft scaffold [1, 2, 25]. However, due to this long-term immunosuppression, the patient is more susceptible to other problems, such as 
opportunistic infections or other systemic effects. Therefore, this technique is only used in the most severe injuries [2, 25].

Allograft is easier to use, as it lacks donor site morbidity and presents unlimited supply and ready accessibility, compared to the autograft [1]. Nevertheless, there are still some problems regarding nerve allograft, leading to the emergence of artificial NGCs to enhance nerve regeneration and eliminate the necessity for immunosuppressants [2]. Given the disadvantages of the technique, new approaches are being developed to achieve better outcomes in PNI.

\subsection{Emerging approaches for axonal fusion}

Photochemical tissue bonding is an emerging technique. It consists in cutting, on either side of a coaptation, the epineurium, followed by staining with a photoactive dye and using laser irradiation, with the purpose to affect a watertight seal across the gap. The use of this method, in a rat model with a sciatic nerve transection, showed that the axonal fiber diameter and myelin thickness improved, in comparison with the traditional suture coaptation $[27,28]$.

Another recent fusion technique, to restore nerve continuity and function, is the use of polyethylene glycol (PEG) to fuse the ends of transected axons. The PEG fusion has to be performed shortly after the injury to have positive effects. It consists in washing both cut ends of the nerve, with calcium-free hypotonic saline, and treating with methylene blue, an antioxidant, at least PEG is applied [27]. PEG works as a fusogen, with the proper alignment of the cut proximal and distal fascicles. Besides, in animal models, this technique demonstrated better results using PEG fusion over epineural suture repair, concerning the motor testing, histology, and other methods to quantificate recuperation $[27,29]$.

\subsection{Non-surgical treatment methods}

Usually, non-surgical and surgical methods are combined, but the success rate in PNI injuries and in long nerve gaps $(>3 \mathrm{~cm})$ is still unclear $[2,30]$.

The beneficial role of exercise on the nervous system is well stablished, promoting axonal growth and phenotypic changes in peripheral nerve architecture [15]. There is an increase of growth factors (stimulation of peripheral nerve regeneration improvement of synaptic elimination and release of neurotrophic factors and help relieve neuropathic pain, by stimulating the opioids release [5]. Aerobic exercise, such as swimming and walking, is the best option to help treat PNI. The volume of training, intensity and the type of nerve injury are parameters that influence the effect of the exercise [15]. The combination of physical exercise with other therapeutic methods, such as electrical stimulation, seems to help achieve better outcomes in the regeneration of PNI [5]. In rats with peroneal nerve injuries, following ten weeks using the treadmill for running at a 10 $\mathrm{m} / \mathrm{min}$ velocity for $1.5 \mathrm{~h}$, two times a day, the fast nerve fibers showed an enhancement in conduction velocity $[15,31]$. In addition, this exercise also reduced the levels of myelinassociated glycoprotein, which is an axonal growth inhibitor, thereby proposing an explanation for the axonal growth stimulated by the exercise [15].

Electrical stimulation (ES) is frequently used as a treatment, since low-frequency electrical stimulation has a positive effect on the nerve regeneration $[1,5,32]$. The combination of ES and the physical activity, like treadmill, also has positive effects [32]. In this technique, it is important that the frequency range is correctly selected, because higher frequencies can deteriorate and exacerbate atrophic muscle events [1, 5]. In addition, it is essential to standardize some factors such as the duration of the electrical 
stimulation, consider the side effects on the healthy surrounding tissues and the variations provoked by the type of injury and extent of damage [1, 32]. To accelerate nerve regeneration, ES can be used in combination with steroids or combined with surgical techniques [1,5]. One study where weekly electric stimulation was applied, using an implanted wireless device, to stimulate rat sciatic nerve regeneration, showed acceleration in the recovery $[27,33]$. Thus, the ability to administer ES under local or regional anaesthesia and using wireless implanted devices would be a promising technological advance and a new approach to common treatments [27].

Another non-surgical method is the magnetic stimulation, activating peripheral nerve regeneration by raising the number and diameter of the regenerated axons to promote functional recovery [5]. This technique is not totally understood but its effect is probably due to the NGF activity stimulation and diminished cytokine activity. The positive effects of this therapeutic method depend on the amplitude $(0.3$ to $300 \mathrm{mT})$, time $(10 \mathrm{~min} /$ day to 24h/day) and frequency of exposure ( $2 \mathrm{~Hz}$ to $2000 \mathrm{~Hz}$ ). Also, in combination with mesenchymal stem cells, it promotes a neuron-like cells differentiation, interfering with the cell cycle [5].

Low-intensity ultrasound (LIU) can induce a positive response in peripheral nerve regeneration, probably through mechanical and thermal effects. The ultrasound promotes membrane diffusion movements and stimulates the blood circulation, the release of brain derived neurotrophic factor (BDNF) and cell's metabolism, as well as, besides stimulating tissue nutrition [5].

Phototherapy, a low power radiation, also promotes axonal regeneration. This technique has positive results, such as diminished scar tissue formation, reduced degenerative process and stimulation of myelination and axonal regrowth [5].

Photobiomodulation therapy (PBMT), using low-level infrared light spectrum lasers, is also considered a therapeutic advance. Laser therapy is considered a non-invasive treatment that activates a photochemical reaction in the cells, resulting in the increase of DNA and RNA synthesis. Consequently, protein synthesis occurs alongside cell proliferation, promoting changes in nerve cell action potentials [32]. The effects are associated with tissue biostimulation. Both in vivo and in vitro studies have shown that this technique enhances the functional recovery and regeneration of the injured peripheral nerve, by stimulating SC proliferation and expanding the axonal diameter [32]. Nevertheless, there are still some issues related to the application parameters, since there is no standardization in treatment with PBMT $[32,34]$.

\subsubsection{Growth factors}

Nerve growth factors (GFs), are molecules released naturally in the injury process and directly enhance nerve regeneration. For that reason, mimicking their release can be an important tool for nerve survival, differentiation, and growth [5, 25, 35]. Therefore, adding these factors is another therapeutic approach can improve the microenvironment of guide tubes, making them more permissive for axonal regrowth [25]. On the other hand, the artificial application of GFs as a therapeutic option is complex, because of their vast biological activity, making it crucial to be administered in small and precise doses. Other limitation is the short biological half-life and the pleiotropic effects. To improve this technique, in addition to a better characterization of the molecule's profile, delivery systems are being investigated [25, 35].

There are diverse GFs and each one has different actions, such as promoting functional regeneration, supporting SC migration and axonal elongation. There are three different groups of molecules with these effects: the neurotrophins (nerve growth factor (NGF), BDNF, neurotrophin-3 and neurotrophin-4), neuropoietic cytokines and the glial-cell-line- 
derived neurotrophic glial-cell derived neurotrophic factor (GDNF), ciliary neurotrophic factor) $[5,25,35]$. From these, NGF is the most explored so far, since it is commonly present in healthy nerves, its expression raises in injured nerves, and has essential functions to enhance neuron growth and survival [5]. GFs biological activity depends on their interaction with some target receptors that are expressed on the neuronal cells surface [25]. The main reason to use these factors is to enhance axonal elongation and SC migration, by providing a neurotrophic and neuroprotective environment, after nerve injury [25]. In addition, GFs can reduce the p38MAPK expression, a pathway activated in early stages of lesion in the PNS and associated with cell death [5].

GFs can be used in combination with NGCs, directly into the conduit lumen or through different carriers [25]. The selection of neurotrophic factors and the suitable delivery method for these molecules are important to achieve good regenerative outcomes. The delivery of GFs from the conduit wall can be achieved with methods like conjugation, adsorption and physical entrapment in the wall. To achieve a beneficial effect on nerve regeneration it is important to consider the optimal dose and GF release kinetics, for standardization and NGCs optimization. Support cells are attracted by the growth factor delivery into the conduit lumen that increases axonal regeneration and contributes to a better microenvironment for repair of nerve gaps [25]. Cristina et al., tested two methods (crosslink and adsorption) and compared their ability for controlled delivery and retention of two different GFs, GDNF and NGF. To assess the outcome of the NGCs delivering the GFs, this study used a $100 \mathrm{~mm}$ sciatic nerve injury model in rats. The results proved that the controlled release of GDNF and NGF from NGCs enhanced nerve repair [36]. Other recent study hypothesized that mineral coated microparticles (MCMs) could generate a sustained release of NGF and GDNF inducing nerve regeneration and a significant improvement in functional recovery. This study used a rat model of the sciatic nerve and the in vivo results in the rats with nerve grafts incorporated with MCMs releasing both the GFs showed significantly more myelinated axons, comparing with the nerve grafts without growth factor treatment [37].

BDNF is a neutrophin that regulates the synaptic function and has potential to enhance nerve regeneration and preservation. In addition, this molecule is important in the reduction of food intake and, consequently, body weight and anorexigenic regulation. For that reason, reducing energy intake has been proved to enhance the neuronal process and the synaptic activity. It has been shown that, in rats with energy limitation, the peripheral nervous structures were markedly preserved, and the degenerative events diminished [38]. Since it is improbable to advise patients to limit their energy uptake and recommend a low-calorie diet, on peripheral nerve injuries, this result does not show a promising therapeutic option.

\subsubsection{Mesenchymal Stem cells}

Following PNI, axonal outgrowth depends on many factors, such as the infiltration of immune cells, neurovascular regeneration and the phenotype of SCs transformation [4]. SCs based therapies have been employed in many studies to enhance nerve regeneration, providing the nutrition and support for axonal growth [4]. Furthermore, over the years, various studies have focused on methods to promote axonal regeneration, without the consequence of sacrificing other healthy functioning nerves, as in the technique of using autologous nerve graft. The need to improve these technique limitations brought the urge to study synthetic nerve conduits, that will be discussed further on, and mesenchymal stem cell - based therapy, which is thought to be a promising strategy to PNI and a number of other diseases $[4,17,18,39,40]$. MSCs can promote regeneration by signaling 
through cell-to-cell contact, cell differentiation into tissue-specific cell types and release of neurotrophic factors [18].

MSCs are multipotent stem cells that can be harvested from numerous sites of the body, such as adipose tissue, dental pulp, bone marrow, umbilical cord, and amniotic fluid [17, 39, 41, 42]. The different tissue origins where MSCs can be harvested explain different characteristics among these cells, such as distinct cytokine expression profiles. Besides, some origins are better than others, to a certain clinical application [39]. In addition, MSCs are undifferentiated, easily isolated, plastic adherent when maintained in standard conditions, and have low immunogenicity. For that reason, MSCs may be transplanted allogenically with minimum consequences [40-42]. Furthermore, MSCs can differentiate into SCs, one of the reasons why these cells are so valuable to PNI [39, 42]. Mesenchymal and Tissue Stem Cell Committee of the International Society for Cellular Therapy human MSCs characteristics by expressing cluster of differentiation CD 105, CD 73 and CD90 and lack of expression of CD 45, CD34, CD 14 or CD 11b, CD79 or CD19 and HLA-DR surface molecules. At least, these cells must differentiate in vitro, in the presence of adequate differentiation media, into osteoblasts, adipocytes and chondroblasts [40]. Progressively, MSCs are considered to act through paracrine effects by the release of extracellular vesicles, which contain components like cytokines and miRNA and can enhance axonal regeneration [39].

In peripheral nerve regeneration, MSCs have an important role due to their ability to produce and release neurotrophic factors that induce axonal growth, their self-renewal capacity, the ability to differentiate into myelinating cell lines and into Schwann-like cells $[17,41,42]$. MSCs have been studied as an important tool for tissue repair because of their capacity to migrate to the site of injury and secrete bioactive factors. This contributes to the paracrine activity, and the ability to suppress the inflammatory response injury, which promote nerve regeneration [42]. Also, MSCs prevent cell death by diminishing the expression of pro-apoptotic factors while increasing anti-apoptotic activities [42]. Some of the multifunctional characteristics of MSCs make them ideal for therapeutic application. For these reasons, in this review, different types of stem cells will be addressed, summarizing the literature supporting MSCs use in PNI.

\subsubsection{Bone marrow mesenchymal stem cells}

Bone marrow mesenchymal stem cells (BM-MSCs) are one of the most studied cell types in this field [18]. These multipotent cells can differentiate into mesenchymal lineages (fat, bone, muscle) but also into non-mesodermal lineages such as astrocytes, neurons and Schwann-like cells, under certain environmental conditions [18, 41, 43, 44]. BM-MSCs produce and secrete neurotrophic factors (NGF, GDNF, ciliary neurotrophic factor and BDNF) and extracellular matrix components (collagen, laminin) that enhance regeneration and angiogenesis [18, 41, 44]. In comparison with other MSCs sources, these cells also have some limitations such as the invasive and painful extraction of bone marrow, some ethical controversies and decreased capacity for proliferation and differentiation $[41,44]$.

Some studies, particularly using the rodent model, found that BM-MSCs application in combination with conduits and nerve grafts showed increased axonal regeneration, improved wet muscle weights and walking track scores [18, 41, 45, 46]. However, BMMSCs have also been studied in larger animal models, such as rabbits and sheep [18]. One recent study tested BM-MSCs, platelet rich plasma with a biodegradable scaffold in nerve gaps with $1 \mathrm{~cm}$ on the sheep radial nerve. Results proved that 6 months later, morphometric and neural conduction improved, showing myelinated nerve fibers both in 
the proximal and distal segments of the nerve when treated with BM-MSCs combined with a scaffold [47].

\subsubsection{Adipose-derived mesenchymal stem cells}

Adipose-derived Mesenchymal stem cells' (Ad-MSCs) source is the stroma of fat tissue, making them accessible and abundant $[43,48]$. In comparison with BM-MSCs, AdMSCs can be collected in a liposuction, a less invasive manner and in higher quantities $[16,48,49]$. Besides, the capability of differentiation and proliferation are much higher than other sources of MSCs [16, 49]. In addition, these cells have low immunogenicity and are able to maintain long-term plasticity and phenotype in vitro [49]. Another important advantage is that location and age of the donor does not influence the therapeutic effect. For these reasons, Ad-MSCs are attractive for regenerative medicine, tissue engineering and peripheral nerve regeneration [48, 49].

These cells follow the criteria of the Mesenchymal and Tissue Stem Cell Committee and can secrete various cytokines, growth factors (VEGF, granulocyte colony-stimulating factor, hepatocyte growth factor) increase myelin sheaths formation, suppress inflammation, guide outgrowing axons and differentiate into a SC phenotype, which indicate multiple advantages for regenerative approaches [16, 43, 48, 49]. The transplantation of canine Ad-MSCs, after crush lesion of the rat sciatic nerve, accelerated functional motor recovery, proved by the results obtained from electromyography and sciatic function index gait analysis, in some studies [16, 43, 48].

\subsubsection{Dental pulp stem cells}

Dental pulp stem cells (DPSCs) are a suitable stem cell source because these cells can be obtained from orthodontically extracted premolar and impacted third molars, which does not raise ethical controversy nor extra harm [44, 50,51]. These MSCs have ectodermal origin and migrated from the neural tube region into the oral region and differentiated $[43,50,52]$. DPSCs are multipotential cells, and when exposed to different differentiation media these cells proved their osteogenic, neurogenic, adipogenic, chondrogenic, dentinogenic and myogenic differentiation capacity, as well as plasticity, self-renewal and high proliferation rate $[43,50,51]$.

These cells express several markers including the mesenchymal (CD13, CD29, CD44 and CD146), specific neuronal markers and BM-MSCs markers [51, 52]. DPSCs ability to differentiate into Schwann-like cells, the capacity to promote myelin reconstitution and axonal regeneration are some of the reasons why these cells can be used in PNIs [44, 50]. In addition, these cells can secrete neurotrophic factors that provide trophic support for a better axonal regeneration and neuroprotection $[43,50]$. After a crush in the sciatic nerve of rats, Okuwa et al., transplanted DPSs at the lesion site. Fourteen days after the surgery, the recovery of motor function was shown through a higher ratio of tibialis muscle weight. The morphological and functional findings proved a good correlation between the use of these cells and peripheral nerve regeneration [53].

\subsubsection{Fetal tissue-derived mesenchymal Stem Cell}

Amniotic fluid and umbilical cord (both considered as fetal tissues) are the most primitive sources of MSCs [44].

The umbilical cord is a relevant source of MSCs, both from umbilical cord blood and the Wharton jelly [18, 44, 54]. So, umbilical cord derived Mesenchymal cells (UC-MSCs) can be harvested in a non-invasive way from postnatal tissue and possess high capacity 
to expand ex vivo $[39,44,54]$. These cells have previously demonstrated the potential to express pluripotent stem cell markers, such as Nanog, Sox 2, ABCG2, Oct4 and the neuroectodermal marker nestin $[18,54]$. Also, UC-MSCs are multipotent stem cells with self-renewal ability and the capacity to differentiate into osteocytes, chondrocytes, adipocytes and neuronal cells [54].

In comparison with other sources of MSCs, these cells have decreased expression of HLA-I and possess greater paracrine effect $[18,39,55]$. In addition, through sequential treatment with B-mecaptoethanol and some cytokines, UC-MSCs can adopt a Schwannlike phenotype. These characteristics make them able to potentiate peripheral nerve and axonal regeneration [39]. Other potential advantages are the low probability of resulting in rejection, the availability off-the shelf and the lack of related ethical considerations [18, 54].

UC-MCSs have been investigated in various studies including in sciatic nerve injury, optic nerve injury and recurrent laryngeal injury, proving to improve recovery and to promote neurogenesis $[18,39,54]$.

Wharton's jelly is an easily accessible, noncontroversial source of MSCs and has unique properties. Also, amniotic mesenchymal stem cells (AMSCs) are relatively low immunogenic cells that derive from the avascular amniotic mesoderm [44, 55]. The collection of amniotic cells can easily be accomplished by prenatal testing and the amniotic membrane can be collected using standard isolation methods after cesarean section [55]. These cell administrations improve functional recovery after nerve injury for different reasons. For instance, AMSCs secrete angiogenic factors, such as VEGF, express chemokine genes and receptors (CCR3, CCR2, CCR5), increase cell migration, endothelial trans differentiation properties and engraftment [44]. Additionally, to the MSCs characteristics, these cells have the capacity to differentiate into neural tissue under specific conditions [44]. Besides, these cells have some advantages, in comparison with other stem cell sources, such as low/no-tumorigenicity, multi-differentiation and renewal potential, no ethical or legal concerns and impressive paracrine effects [56].

\subsubsection{Skeletal-muscle derived stem cells}

Skeletal muscle-derived stem cells (Sk-SCs) can be obtained from skeletal muscle satellite cells and have the capacity to differentiate into various lineages such as adipogenic, osteoblastic, neuronal, myogenic and glial [18,44]. These cells represent an opportunity in peripheral nerve regeneration. In a study of long nerve gap injury in a murine model, Sk-SCs differentiated into Schwan cells and improved the endoneurial and perineurial architecture, which suggests the ability to reconstruct the muscle-nerve-blood vessel unit $[18,44]$.

Muscle progenitor cells (MPC), have well-characterized markers and are an easily accessible type of cells, simple to manipulate in culture. Reut et al,. evaluated the use of MPC overexpressing the NTF genes in a mouse model of sciatic injury, and proved that the combination of these cells with such important trophic factors enhance the peripheral nerve regeneration $[23,57]$.

\subsubsection{Hair follicle-associated pluripotent cells}

Hair follicle-associated pluripotent (HAP) cells are in the hair follicle, express nestin and are being studied because of their involvement in the formation of the hair follicle sensory nerves [18]. These cells can differentiate into glial, neuronal lineage, smooth muscle myocytes, melanocytes, keratinocytes and are considered pluripotent. The 
problem concerning the use of HAP is that they remain pluripotent in regenerative models, with neuroglial differentiation making it difficult to assure a specific differentiation into one of the possible lineages [18].

\subsubsection{Neural Crest Stem Cells}

Neural crest stem cells (NCCs) have an important role during neurogenesis, especially in the development of the spinal cord and brain, which occurs during embryogenesis [41]. In the human adult, these cells are located in the subventricular regions and in the hippocampus. These cells maintain their multipotency and can differentiate into astrocytes, neurons and oligodendrocytes [18, 43]. Also, these cells have already been identified in both postnatal adult and embryonic tissues, which include the bone marrow, cornea gut, heart, sciatic nerve and also the skin [18]. Transplanted neural stem cells can differentiate into neurons and Schwann-like in injured peripheral nerves, secrete BDNF, fibroblast growth factor, insulin-like growth factor and NGF, restore angiogenesis, form myelin and enhance nerve growth [16].

For those reasons, some studies suggest that the application of NCCs in the injury site, both after acute and chronic PNI, can enhance nerve regeneration. After sciatic nerve injury, one study used diverse methods to quantify the result of applying NCCs transplantation in the lesion site. These cells showed a great potential in regeneration potency, especially at the fifth passage [23]. However, the limitations of these cells include the technical difficulties associated with cell harvest, tumorigenic risks and the urge to differentiate directly into specialized neural cell lines [18, 41, 43].

\subsubsection{Skin-derived precursor cells}

Skin-derived precursor cells (SKPs) originate in dermal papilla and have the ability to differentiate into neurons, glia and smooth muscle cells. These cells have already proved to be beneficial in sciatic nerve repair and in the ability to improve regeneration after chronic denervation [18].

\subsubsection{Olfactory mucosa mesenchymal stem cell}

Olfactory mucosa mesenchymal stem cells (OM-MSCS) are collected from the olfactory mucosa, which anatomical location is ideal for both postmortem and antemortem collection in various species of clinical interest. These cells have fibroblast-like morphology, clear plastic adhesion and express specific surface markers with no expression of hematopoietic markers, as expected from MSCs. In addition, OM-MSCs can generate new fibroblast colonies from single cells and are able to differentiate towards adipogenic, chondrogenic, osteogenic and neurogenic cell lines [22, 58].

A recent study used chitosan NGCs and OM-MSCs in different therapeutic approaches to enhance rat sciatic nerve injury, following neurotmesis. All the therapeutic groups receiving chitosan NGCs and the cells had best final results in the functional assessment, kinematic analysis and histomorphometric evaluations, 20 weeks after the inflicted injury. These results demonstrate promising effects of the use of OM-MSCs in PNI [59].

\subsubsection{Secretome}

All the previous mentioned MSCs can secrete bio-active factors, defined as secretome that comprises chemokines, cell adhesion molecules, cytokines, lipid mediators, growth factors, hormones, micro vesicles and so on. The constituents are considered the main 
participants in tissue repair and regeneration, through their paracrine capacity that mediates cell-to-cell signaling [42, 49, 60]. The secretome involves two components: a vesicular fraction composed by different types of vesicles, crucial in the delivery of microRNAs and proteins, and a soluble fraction constituted by cytokines and chemokines [60]. For those reasons, recent studies reinforce the effectiveness of MSCs secretome therapeutic potential in different diseases [42, 49].

This new line of research contributes with some advantages over cell-based applications. Secretome has lower immunogenicity, compared to living and proliferating cells and the analysis of secretome safety, dosage and potency is simpler [49, 60]. The transcriptome and proteome are very different in MSCs populations, so the secretome profile is also heterogeneous [42]. The use of various types of MSCs and methods to develop secretome, for the treatment of various diseases, including PNI, are receiving more attention in recent time [61]. Amir et al., incorporated on a 3D-polycaprolactone scaffold the BM-MSCs' secretome to examine the efficiency in an axotomy in the rat model. In this study there were four groups, one control, the second with just the axotomy (10 $\mathrm{mm}$ of the nerve was removed), the third with axotomy and only the scaffold and the last with the scaffold incorporated with the BM-MSCs' secretome. After 12 weeks of treatment, the results showed that the sciatic nerve was significantly restored, especially in the third and fourth group. In addition to the advantages from using secretome, in this group the levels of NGF and BDNF were increased [62].

Other recent study used Schwann-like cells derived from differentiated human embryonic stem cell-derived neural stem cells secretome to explore the influence in angiogenesis and nerve regeneration. After sciatic nerve crush injury, using the rat model, this secretome was used to study its role in nerve regeneration. In comparison with the control group, the animals treated with the secretome showed better gait recovery and the gastrocnemius wet weight was higher, after 14 days [63]. Martins et al., investigated the UC's secretome on axonal elongation of CNS neurons. The results proved that this secretome promotes axonal outgrowth in primary cultures of rat embryonic hippocampal and cortical neurons [64].

\subsubsection{Gene therapies}

In recent years, it was noticed that some alterations in the genes (insertion, alteration or removal), in some cells, can be used to treat diseases [5].

The modulation of regulatory proteins and neurothrophic factors can enhance postsurgical results. The main goal of gene therapy in PNI is to increase axon regeneration and the most efficient technique for nerve regeneration is gene delivery [5]. For instance, injections of a viral vector (every $5-8 \mathrm{~mm}$ ) has been proved to be highly successful in the gene transduction of the rat's sciatic nerve [65]. In addition, this technique can be efficiently performed at the site of repair. Afterwards, retrograde transport of the viral vectors distributes it to the cell body, a place where gene transcription may be changed [27]. Animal models were successfully transfected with genes encoding neurotrophic factors, and the recovery achieved more rapidly. Besides, SC is another target for gene therapy that has shown improvement in regeneration. The use of ciliary neurotrophic factor, a factor unique to the SC, in a mouse model of sciatic nerve crush injury, was transduced using an adenoviral-associated vector that demonstrated an enhancement in axonal regeneration $[27,66]$. Another hypothesis, concerning its use is the overexpression of c-Jun, a transcription master switch which is upregulated in case of an injury [27]. In short, gene therapy is a promising method for the customizable delivery of neurotrophic factors, but there is still a long way to go for this technique to be fully effective and safe $[5,27]$. 


\subsubsection{Pharmacological}

To avoid scar formation in peripheral regeneration barriers can be surgically created between the surrounding tissue and the injured nerve, complemented by pharmacological agents as steroid hormones [5]. They can promote myelination and have neuroprotective proprieties [32].

Corticosteroids are also a possible treatment option for nerve injury. The study of Bernstein et al., demonstrated that the administration of oral corticosteroids enhances the motor and sensory recovery after iatrogenic PNI $[32,67]$. These substances act inhibiting fibroblast growth and the migration/phagocytic action of granulocytes, selectively [5]. For example, dexamethasone, frequently used to reduce edema and the neural inflammation effects, turns up to enhance nerve recovery after injury [5]. The animals treated with these substances are associated with higher recovery rates $[5,32]$. Thus, the use of hormonal therapies is not commonly used in the treatment of PNI because these substances can have side effects.

Erythropoietin (EPO) is an endogenous hormone, a hematopoietic agent, especially used in the treatment of anemia. EPO has shown neuroprotective properties both in CNS and PNS. In addition, EPO and its receptor are present in a variety of non-erythroid cells, and for that reason have an impact in many biological functions throughout the body [32]. For instance, EPO is secreted and produced by diverse parts, like neurons of hippocampus, internal capsule, cortex and midbrain. Besides, the receptor is also expressed in the radicular nerves in the PNS on the myelin sheath. For those reasons, EPO is demonstrated to have a positive effect in the sciatic function index of rats and mice, after crush injury $[32,68]$. Besides, the effects were observed in different times of use in the treatment. The mechanism of action is not well known but it is thought that EPO promotes the expression of myelin genes, such as MPZ and PMP22, maintaining more myelinated axons at the site of injury [32].

4 -aminopyridine (4-AP) is used in the symptomatic treatment of multiple sclerosis. It is a broad-spectrum potassium channel blocker, capable of improving neuromuscular function in patients with other demyelinating disorders [5]. The mechanism of action results in the calcium influx that stimulates the release of the neurotransmitter thus causing synaptic transmission and, ultimately, direct effects on the muscle [32]. The capacity of 4-AP increasing cell-membrane excitability and, consequently, impulse condition shows that, relevant doses of this molecule shortly after injury, enhance global function recovery, improve nerve velocity of conduction, allowed a better and faster behavioral and motor recovery and promotes remyelination, in established mouse model after PNI $[5,32,69]$. Besides, 4-AP appears to be axonoprotective and myeloprotective, which can be helpful and contribute therapeutically to recovery due to excitatory molecules that can stabilize impulse conduction [32]. Nevertheless, there are some side effects, and more studies are needed to guarantee the safer use of this substance.

\subsubsection{Nutritional therapy}

Some nutrients can have a role in preserving nerve health and function and enhance the recovery of an injured nerve. Nevertheless, there are several studies about the mechanisms, by which, both nutritional factors and nutrients have an impact [38].

The nervous system is extremely rich in lipids that are fundamental for several functions. Poly-unsaturated acids (PUFA) are recommended to be balanced in each individual diet. For example, a study in Wister rats, proved that the intake of n-3 PUFAs has positive effects on the regeneration of a sciatic nerve injury $[38,70]$. 
It has been proved that vitamins and minerals have a fundamental role in the prevention of oxidative stress, reducing neuroinflammation and providing protection against cellular injury [5]. In addition, these molecules may have helped in the production of endogenous neurotrophic factors, like BDNF, that will enhance nerve repair [38].

Vitamin $\mathrm{B}_{6}$ supplementation mitigates symptoms of neuropathy and numbness. Vitamin $B_{12}$ enhances the number of SCs and promotes axonal regeneration by improving the re-myelination $[5,38]$. Furthermore, there are studies proving that high doses of this vitamin have potential to improve the PNI treatment, in a rat sciatic nerve injury model and that this vitamin can upregulate genes related to various GFs [5, 71]. Other promising result in rats, was the local implantation of methylcobalamin in nanofiber layers. The results demonstrated a positive synergistic effect, where both motor and sensory functions improved, with an enhancement of nerve conduction velocity and myelination, after sciatic nerve injury $[38,72]$.

Ascorbic acid (Vitamin C) helps SC myelination of peripheral axons, both in vivo and in vitro, since co-cultures fail to produce myelinated segments without vitamin C [73]. Tyler et al., using a mouse model, showed that dietary vitamin $C$ deficiency impaired, in early stages, the peripheral myelination [73]. Besides, this study investigated that the intake of this vitamin was fundamental for the expression of periaxin and myelin basic protein, both important components of the myelin sheath. For those reasons, vitamin $\mathrm{C}$ can promote SC myelination, not only by collagen stability but also through direct epigenetic regulation, making this substance critical throughout development and during remyelination, after PNI [73].

Folic acid and even minerals play a role in cognitive functions and enhance neurogenesis. Moreover, phenolic components, such as curcumin, are potent neuroprotectants. Curcumin effect in various dosages on sciatic nerve regeneration has been investigated, demonstrating that a higher dose has better results in the recovery of nerve function and enhanced the re-myelination [5, 38]. Other substances, such as resveratrol, Alpinia Oxyphylla (important herb), epigallocatechin-3-gallate (catechin of green tea), therapeutic effects on PNI are being studied and showing positive effects [38].

Medicinal plants, such as Achyranthes bidentata, Astragalus membranaceus, Panax ginseng, Hericium erinaceus, Acorus calamus, Curcuma longa and Ginkgo biloba have proved ameliorating effects in neuropathic pain [74].

Another important factor for neuroprotection is the early application of antioxidant therapies. For instance, the use of alpha-lipoic acid demonstrated positive effects, as this thioctic acid stimulates an increase in endogenous antioxidants (SOD and CAT). Therefore, alpha lipoic acid can be considered in the treatment of PNIs [5, 38].

\subsection{Nerve Guide conduits}

Other therapeutic option is the use of NGCs. NGCs are tissue engineered tubular structures that serve as bridge between the proximal and distal ends of the injured nerve $[1,2,12,43]$. These can be made of natural and/or synthetic biopolymers, which are planned to possess the necessary biomechanical and mechanical cues for neural regeneration [1]. Most of the disadvantages described regarding the use of autografts can be avoided by the use of NGCs [1, 11]. NGCs allow trophic and structural support for both the nerve ends, supporting the regrowth of axons alongside the conduit and the invasion of the surrounding tissues. Also, these conduits can have neurotrophic factors, proteins and even extracellular matrices, throughout their inner surface, that can increase the axon regeneration [1, 2, 43]. Besides, the most valuable improvement of a conduit is its capability to provide a better microenvironment for neuronal recovery. For peripheral nerve repair, there is a need for compliance between support cells, conduits, growth 
factors and physical stimuli [43]. Therefore, NGCs act as a guide for nerve regeneration, since they allow cells to adhere and migrate, thus achieving the desirable regeneration [43].

An ideal NGC has some requirements, including structural features to longitudinally align the regeneration axons, biomimetic architecture, mechanical properties to provide structural support, adequate permeability for trophic support, wall thickness, specific conduit diameter, compliance, neuro inductivity, low toxicity, electrical conductivity, biodegradability, biocompatibility and flexibility [1, 2, 43, 75]. In 1982, the first application of NGCs was made, using non-resorbable silicon tubes to repair a $6 \mathrm{~mm}$ nerve gap. Since then, researchers are trying to obtain the ideal NGC, improving the design, fabrication process and materials, especially for complex defects [1, 2]. Different artificial nerve conduits have already been studied and are commercially available and approved by the Food and Drug Administration and European Medicine Agency, especially for short nerve lesions $[43,75]$.

After manufacturing, the biomaterial is tested in vitro models with the purpose to access its cytotoxicity, biocompatibility, genotoxicity, degradation, cell proliferation and interaction between cells and the biomaterial [75]. The use of in vitro assays is crucial for the study, making it more easily standardized and reproducible and aids reducing the number of animals used in the in vivo testes taking into consideration the " $3 R^{\prime}$ " philosophy (Reduce, Replace, Refine) [75].

Kornfeld et al., recently used spider silk as a potential material of choice to overcome the limitations of nerve gap repair of $3 \mathrm{~cm}$. This material has been proved to be an effective guidance architecture for SCs and to allow nerve regeneration both in rodents and ovine models. One recent study examined the time course of axonal regeneration rate using spider silk nerve implants and compared to autologous nerve grafts in a $6.0 \mathrm{~cm}$ nerve defect in adult sheep [30]. The results obtained proved that the conduits were as effective as the autologous nerve implants, without the potential morbidity of autologous nerve grafts (sensory loss, neuroma formation) [30].

\subsubsection{Transplantation of cell-seeded nerve conduits - peripheral nerve regeneration}

The previously mentioned MSCs-based therapies can be used in association with NGCs, to promote nerve regeneration. The successful outcome of this therapy depends on the production of extracellular matrix proteins and trophic factors, to create a favorable environment for axonal outgrowth. In addition, the differentiation into SC lineage benefit myelination of regenerating axons [43]. Numerous types of cells, previously described, can be applied inside NGCs. It is important to choose the NGCs depending on the therapeutic option in study. For instance, the structure is fundamental for the cells to enter and proliferate when seeding MSCs in NGCs.

One recent study used canine Ad-MSCs in 3D- printed polycaprolactone NGCs in a sciatic rat model, $12 \mathrm{~mm}$ gap injury. In addition, a scaffold composed of heterologous fibrin biopolymer derived from snake venom was used to retain Ad-MSCs into the internal wall of the NGC. This material is non-cytotoxic, biodegradable and the sealant properties are perfect to avoid Ad-MSCs loses from the NGCs. The positive functional and electrophysiological locomotor recovery, after 8 and 12 weeks, proved that different combinations can be a desirable approach to overcome PNI [76].

Jahromi et al., developed a novel strategy for PNI with combination of gold nanoparticles and BDNF - encapsulated chitosan in lamini-coated nanofiber of poly(lactide-co-glycolide) (PLGA) with rat Ad-MSCs suspended in alginate. In this study, alginate was used as a hydrogel to retain the Ad-MSCs, providing an environment that 
prevents the leak out of the cells. This therapeutic technique was tested in a rat sciatic model and the results showed that this complex NGCs could enhance nerve regeneration [77].

Recently, a group developed a tissue-engineered NGC coated with induced pluripotent stem cells - derived neurospheres in a rat sciatic nerve model, $5 \mathrm{~mm}$ injury. This study had three different groups, one with the aforementioned therapeutic option, the control and other with the autograft. The results from the autograft were slightly better than the therapeutic option. However, the group with the cells showed higher axon number and good outcomes making this other promising cell-based therapy using NGCs in the PNI treatment [78].

Bagher et al., loaded different concentrations of Hesperidin, that is a natural bioflavonoid, into cross-linked alginate/chitosan hydrogel and evaluated its characteristics. Following the cytocompatibility tests the achieved hydrogel was administrated in a sciatic nerve injury in a rat. Results from weight loss, walking-footprint analysis, hot plate latency teste and other proved that the NGC loaded with hesperidin improved sciatic nerve regeneration [79]. Thus, recent studies show that NGCs combined with other substances can significantly improve nerve regeneration, and are therefore promising in the treatment of PNI.

\subsubsection{Advances and next generation nerve conduits}

There are some conventional methods used for accomplishing hollow NGCs, including injection molding, melt extrusion, braiding and crosslink. In all these techniques there is a limitation, the generation of simple macroscale conduit structures, with poor geometric accuracy. On the other hand, advanced techniques such as inkjet printing, 3D printing, fused deposition modeling, electrospinning and three-dimensional bioplotting have been investigated to fabricate complex NGCs [25]. Electrospinning is a commonly used method to fabricate nano- or microfibers. It can mimic the natural extracellular matrix on a fiber structure, optimize and manipulate mechanical, biological and kinetic properties, in order to enhance cell-substrate interactions [25].

NGCs engineering achievements can help to guide the axons regeneration and incorporate supportive cells, genetic manipulators, GFs and electro conduction [12]. The traditionally available conduits are simple hollow tubular constructs that do not possess the attributes of an autograft. Recently, major studies have been made directly to design scaffold characteristics to improve the nerve guides efficiency [25]. The different types of possible alterations implicate the addition of luminal fillers as guidance arrangement, neurotrophic factors, supportive cells and conductive polymers inside the tube, with the purpose to improve the efficiency of regeneration, in both small and large gaps [25].

Recently, technology involving 3D printing helps the fabrication of customized NGCs. $3 \mathrm{D}$ printing has advanced the field of regeneration by reproducing a 3D structure that mimics the natural extracellular matrix. However, there are some limitations such as low printing resolution, limited speed of printing and lack of a dynamic environment. For that reason, $4 \mathrm{D}$ printing is one of the most progressive methods of NGCs fabrication for the next generation of NGCs [25]. 4D printing technology can help to fabricate structures that can transform their shape, called the shape-morphing effect [80]. For that reason, these materials can transform and recover its shape depending on the external stimuli (light, temperature and humidity) mimicking the physiological changes in the body [80]. 


\section{In vitro and animal models}

\subsection{In vitro models}

Diverse distinct factors of the peripheral nerve regeneration mechanisms can be reproduced in vitro [81]. In the design of a study, it is important to begin with the in vitro models, before trying to prove those concepts in in vivo models, mainly respecting the 3Rs. For these studies, cell line-based models can be used without any ethical impact.

Cell lines have clear ethical advantages since they replace any further animal use. In addition, these cells can be derived from human tissues, which enhances the translational research perspective towards the clinic [81]. In peripheral nerve research, cells cannot be used to anticipate the in vivo behavior of neurons and SCs because of the structural and anatomical complexity of the organs involved. The limited in vitro reproduction of the in vivo mechanism is the biggest limitation and the real need to use in vivo models.

Organotypic cultures are in vitro culturing conditions that simulate the $3 \mathrm{D}$ architecture of a tissue and/or organ. So, this model has more correlation to the in vivo chemicophysical environment, which contributes to SC and neuron regulation, than the in vitro model based on just cell lines [81]. The main disadvantage, in comparison with the in vivo model of peripheral regeneration, is the limited time space provided for observation of regeneration. Cultures do not survive for months and resemble reconnection to target tissues. Therefore, these methods are fundamental to test a hypothesis and to be aware of the number of animals used but are not enough to have a fundamental experiment [81].

\subsection{Animal models}

In PNI research, diverse animal models are commonly used. Rodents, mainly rats, are most frequently used as models of nerve injury following surgical repair or other treatments in study, to assess the regeneration potency [53]. The choice of animal model depends on many factors, such as availability, easy care, specialized team to perform all the necessary procedures, lowest risk of infection and tolerance to manipulation [43]. Other advantage is that functional outcomes like sensory, motor and sympathetic recovery can be measured to demonstrate the outcome after PNI. To standardize the protocol, it is fundamental that the experimental injury, repair and monitorization, are carefully controlled and described. In addition, these models can provide more results, because at the end point, tissues can be harvested and analyzed. Normally, injured models involve damage of a nerve, followed by random animal aggrupation into test and control groups [53].

The use of rats, as an animal model, has some advantages, such as their resistance to infections and surgery complications, the availability of different strains, the well-studied morphological anatomy and low maintenance costs. Nevertheless, the small size of the rat can also be a limitation because the studies are performed in nerve gaps $<10 \mathrm{~mm}$. However, in humans the damage commonly exceeds $10 \mathrm{~mm}$ and nerve regeneration is slower [5, 43]. Rodents' metabolic rate is higher, in comparison with humans, so nerve regeneration is also faster which makes it difficult to understand the true effectiveness of the used therapies enhancing nerve repair [5, 82, 83]. These rodents' extremely high neuroregenerative ability, makes it harder to correlate to the human species [5, 43].

To overcome the nerve gap size limitation other larger animal models are used, such as rabbits, mini-pigs, dogs, cats and sheep. The rabbit model can be used to study larger nerve gaps (> $50 \mathrm{~mm}$ ), but there are more anatomical changes between these species and 
the human. Dogs and cats can be used, but the ethical issues regarding the use of this species has gradually reduced their use $[5,43]$. The sheep is commonly used due to the similarity between limb nerves [43]. Large models are the only solution to replicate the regeneration and effects of injuries in large nerve defects. Recently, an alternative model using the sheep was established. In this model, neurotmesis and axonotmesis injuries in the common peroneal nerve were induced. Afterwards, different therapeutic methods were applied to standardize the use of the sheep in nerve regeneration studies. To evaluate the nerve regeneration, a neurological examination protocol was established and adapted to the common peroneal nerve [83].

The zebrafish is another popular model that is used in several fields of regenerative medicine. This animal has a long superficial peripheral nerve in the posterior lateral line, and this line possesses the common cell types present in the PNS. Laser-mediated nerve transection coupled to time-lapse confocal microscopy is an advanced methodology that helps to investigate the degeneration and regeneration process of peripheral nerves in the zebrafish [20].

\section{Quantifying regeneration following peripheral nerve injury}

\subsection{Methods to measure nerve recovery in animals}

Commonly with PNI gait changes occur. The previously described animal model is of utmost importance since the details of animal limb movements during locomotion help in the PNI assessment [23]. Various functional tests can aid in the motor recovery evaluation after PNI. These methods are fundamental to understand the therapeutic potential of some therapies [43]. As explained throughout this review, it has not yet been possible to select a therapeutic approach that has unequivocal efficacy. With these methods the optimal treatment, for each type of nerve injury, can be optimized and supported by scientific results. The methods are chosen depending on the study, cost, time to conduct the experience and technical difficulty.

Concerning motor recovery, the majority of the tests are noninvasive and simple. Besides, they can be executed and help the monitorization, throughout the recovery period being very important to fully understand nerve regeneration [53]. One of the methods to measure motor recovery using a functional index score, is the walking track/Sciatic Functional Index (SFI) [23]. In this technique, the paw placement is monitored and also recorded to evaluate the animals' walking, throughout a narrow corridor. It is a non-invasive method and provides access to the functional recovery of the hindlimb by recording and observing the footprint, considering the hindlimb as a whole. To perform this evaluation method the animal is placed inside a transparent acrylic corridor and bellow there is an image capture installed. The animal is placed in the beginning of the tunnel and to stimulate the animal to walk through the corridor there is a shelter in the end to encourage the animal to walk in that direction (140). Different parameters are analyzed so that it is possible to obtain the SFI $[43,53]$. An SFI of 0 is normal and an SFI of 100 indicates total impairment of the sciatic nerve [43]. The main limitations are the dragging of the tail during the footprint record, the development of flexion contracture and smearing of the paws [5]. Basso, Beattie and Bresnaha is a classic behavior method, which consists in the observation of a paralyzed rodent for 4 minutes in an open area. It is based on the assessment of some functional behaviors, like the paw placement and the limb movement. After, the locomotion the method allows to achieve a score from 0 (no observable locomotor movements) to 21 points (normal locomotor movements) [5, 23, 43].

The withdrawal reflex latency (WRL) is used to determine the integrity of nociceptive function. The animal is wrapped in a soft sloth and suspended over a heating plate at $56^{\circ}$ 
temperature. WRL is defined by the time, in seconds, that the animal requires to retract its hind limb after the contact with the plate [59].

Another assessment, called Static Sciatic Index (SSI), is obtained by the foot muscle function analysis. Which is basically performed with the animal in stationary position, to measure the toe spread [43]. The animal is placed inside a transparent acrylic box, placed inside the corridor. With both limbs in contact with the box floor this moment is caught in a photography bellow the acrylic box [59]. One innovative method is the use of an automated gait analysis system, CatWalk XT. This consists in a glass walking platform, recorded by a high-speed digital camera, illuminated by green light and the software is recording, analyzing and processing the data collected from rodent paw prints. This method can overcome the human error of observation, because it uses specialized software to objectively measure several factors of gait. Besides, with the system it is easier to understand subtle gait changes and offers more parameters (associated to individual paw prints, paw prints position and coordination) to analyze [23]. Other described technique used animals walking on an acrylic track with $120 \mathrm{~cm}$ length, $12 \mathrm{~cm}$ width and $15 \mathrm{~cm}$ height. Following trichotomy three reflective markers with $2 \mathrm{~mm}$ diameter were allocated to three different bony prominences on the desired hind limb. Then, a Visual 3D software provided the necessary measurements to determine the animal's ankle angle [59]. Specially in PNIs, these methods can provide information about the upper and lower extremities, such as axonotmesis, neurotmesis or fibrosis [23].

The whisker motion recovery after facial nerve injury, or muscle strength recovery after limb nerve recovery consists in recording animals for 3-5 minutes. Then, analyzes of the angle, protraction, amplitude of retraction and whisking frequency are evaluated, so it is considered a subjective evaluation $[23,43,53]$.

The Von Frey test is performed with the animal placed on a grid and the plantar skin is stimulated, by a noxious mechanical stimulus, using filaments. The purpose is to increase force until the animal responds by paw withdrawal. For comparison, both hind limb nerves are bilaterally evaluated, injured and noninjured site $[43,53]$. There are other methods, like the hot and cold response and the grasping test, that evaluate sensory function [53].

The number of animals can be reduced, if multiple non or minimally invasive functional tests are performed and compared to postmortem assessments. These techniques to assess axonal regeneration include histological analysis of the harvested tissue, immunohistochemistry and retrograde/anterograde tracing [53]. Normally, histology is used to understand the number of axons in a repaired nerve. The different staining provides various information about the nerve regeneration, including the myelin, collagen fibers, cells and non-neuronal cells such as SCs and macrophages [43, 53]. Another useful measure, indicative of the innervation degree, is provided by the muscle distal to the nerve, that can be collected and weighed [53]. Furthermore, these results can be compared with the contralateral healthy side.

Neurophysiology is the stimulation of the nerve using electrical pulses [43]. To obtain these values, the electrodes are allocated proximal to the injury, under anesthesia. There are two possible options, the nerve and muscles can be exposed, and the electrodes allocated directly or, less invasively, with the aid of percutaneous needle electrodes [43, 53]. The amplitude is related to the quantity of functionally reinnervated muscle nerve fibers and to estimate axonal loss. As an alternative, motor unit number estimation, is another potential method to quantify the number of motor axons of the muscle being studied. To provide even more detailed values, compound nerve action potential, which means the record between two positions of the nerve, gives information about conduction in that specific segment under study $[43,53]$. 


\section{Conclusions}

PNI is a condition that affects the quality of life of many people and leads to motor and sensory deficits (19). The positive point is that the PNS has some regeneration capacity, and the minor lesions can heal with full, or almost full, functional recovery in some cases (16). However, as explained in this review, there are more severe lesions that frequently yield unsatisfactory outcomes, even with the gold standard methods of treatment. These severe injuries require treatment, mainly surgery, to help reconnecting the viable extremities of the nerve. In addition, when the nerve gap is bigger there are other options, as the nerve grafts and NGC. Recently, there is a lot of ongoing investigation and efforts to develop new treatment approaches, as new surgical repairs, next generation NGCs and other techniques, such as nutritional therapy, gene therapy and the use of regenerative medicine. There are plenty of different MSCs that can enhance peripheral nerve regeneration, as well as, their secretome. Even though these efforts to identify promising treatments, it is very difficult to achieve a perfect technique that helps diminish neuropathic pain and helps recovering the complete function of the nerve.

The in vitro and in vivo models were explained, as well as, the methods to measure nerve recovery in animals, which are fundamental to understand the true meaning of a study concerning a new approach in the PNI. Besides, in a One Health approach, the translation of these models is crucial, even though there are some limitations. To sum up, more studies are required to achieve better therapeutic options that can provide better outcomes for PNI.

Author Contributions: All authors had made substantial contributions to the work, with wellestablished division of tasks. All authors reviewed the final work and approved its submission. All authors agreed to be personally accountable for the author's own contributions and for ensuring that questions related to the accuracy or integrity of any part of the work, even those in which the author was not personally involved, are appropriately investigated, resolved, and documented in the literature. All authors have read and agreed to the published version of the manuscript.

Funding: This research was funded by Projects PEst-OE/AGR/UI0211/2011 from FCT, and COMPETE 2020, from ANI-Projetos ID\&T Empresas em Copromoção, by the project "insitu.Biomas-Reinvent biomanufacturing systems by using an usability approach for in situ clinic temporary implants fabrication" with the reference POCI-01-0247-FEDER-017771, by the project "Print-on-Organs-Engineering bioinks and processes for direct printing on organs" with the reference POCI-01-0247-FEDER-033877, by the project "Bone2Move-Development of "in vivo" experimental techniques and modelling methodologies for the evaluation of $4 \mathrm{D}$ scaffolds for bone defect in sheep model: an integrative research approach" with the reference POCI-01-0145-FEDER031146 and by the PhD scholarships Mariana Vieira Branquinho (SFRH/BD/146172/2019), Ana Catarina Sousa (SFRH/BD/146689/2019) and Bruna Lopes (2021.05265.BD).

Informed Consent Statement: Informed consent was obtained from all subjects involved in this study.

Acknowledgments: Rui Damásio Alvites (SFRH/BD/116118/2016), Mariana Vieira Branquinho (SFRH/BD/146172/2019), Ana Catarina Sousa (SFRH/BD/146689/2019) and Bruna Lopes (2021.05265.BD) acknowledge FCT, for financial support.

Conflicts of Interest: The authors declare that there are no conflicts of interest regarding the publication of this article.

\section{Abbreviations:}

Ad-MSCs - Adipose-Derived Mesenchymal Stem Cells

ALG - Aligned Chitosan Fiber Hydrogel

AMSCs - Amniotic Mesenchymal Stem Cells

BDNF - Brain Derived Neurotrophic Factor

BM-MSCs - Bone Marrow Mesenchymal Stem Cells 


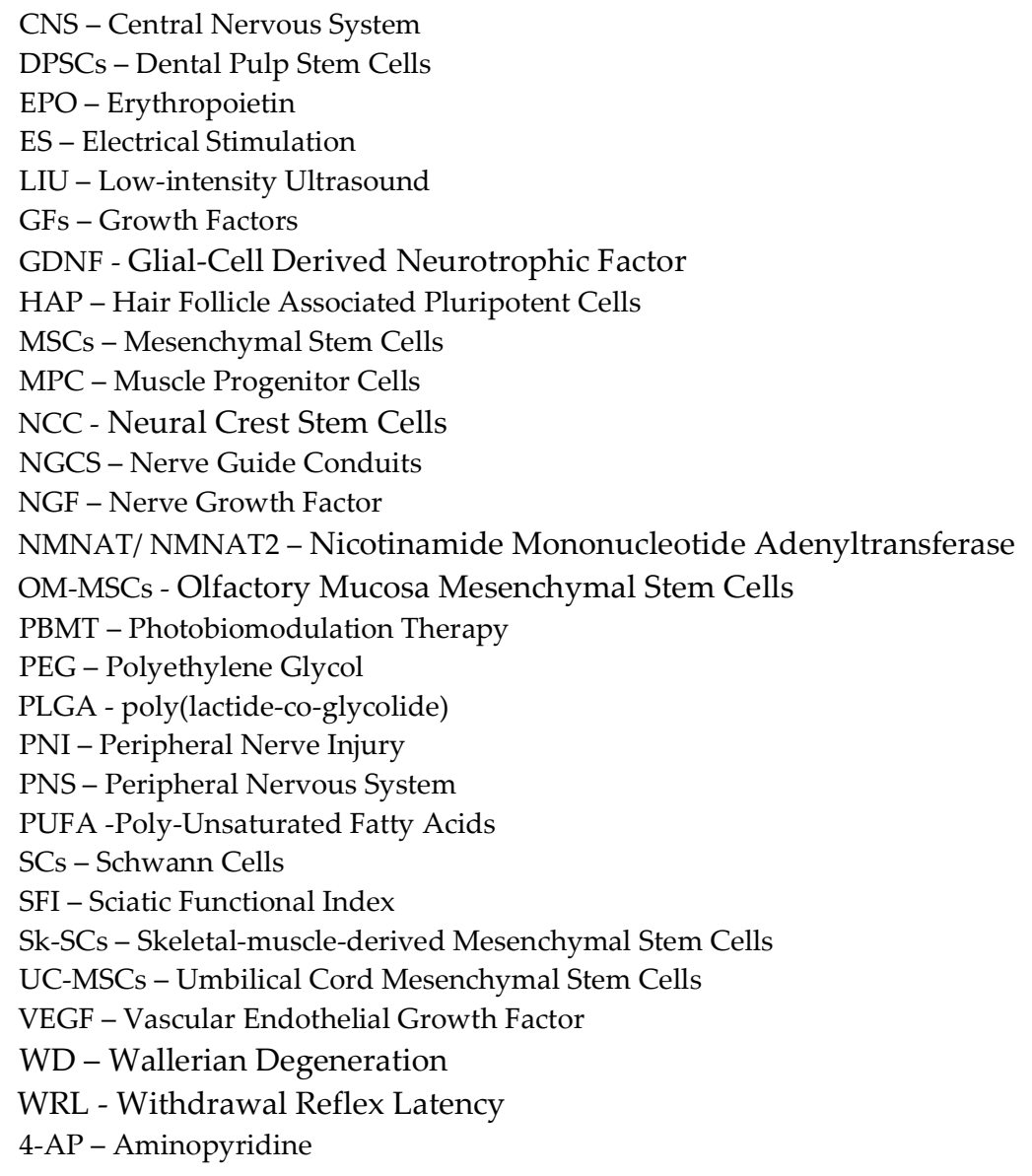

\section{References}

1. Vijayavenkataraman, S., Nerve guide conduits for peripheral nerve injury repair: A review on design, materials and fabrication methods. Acta Biomater, 2020. 106: p. 54-69.

2. Hussain, G., et al., Current Status of Therapeutic Approaches against Peripheral Nerve Injuries: A Detailed Story from Injury to Recovery. Int J Biol Sci, 2020. 16(1): p. 116-134.

3. Zhang, M., et al., Repair of Peripheral Nerve Injury Using Hydrogels Based on Self-Assembled Peptides. Gels, 2021. $7(4)$.

4. $\quad$ Dong, R., et al., MSC-Derived Exosomes-Based Therapy for Peripheral Nerve Injury: A Novel Therapeutic Strategy. Biomed Res Int, 2019. 2019: p. 6458237.

5. Alvites, R., et al., Peripheral nerve injury and axonotmesis: State of the art and recent advances. Cogent Medicine, 2018. 5(1).

6. $\quad$ Wojtkiewicz, D.M., et al., Social impact of peripheral nerve injuries. Hand (N Y), 2015. 10(2): p. 161-7.

7. Szok, D., et al., Therapeutic Approaches for Peripheral and Central Neuropathic Pain. Behav Neurol, 2019. 2019: p. 8685954.

8. Kornfeld, T., P.M. Vogt, and C. Radtke, Nerve grafting for peripheral nerve injuries with extended defect sizes. Wien Med Wochenschr, 2019. 169(9-10): p. 240-251.

9. Wang, M.L., et al., Peripheral nerve injury, scarring, and recovery. Connect Tissue Res, 2019. 60(1): p. 3-9.

10. Slavin, B.R., et al., Insulin-Like Growth Factor-1: A Promising Therapeutic Target for Peripheral Nerve Injury. Front Bioeng Biotechnol, 2021. 9: p. 695850. 
11. Vijayavenkataraman, S., et al., Electrohydrodynamic Jet 3D Printed Nerve Guide Conduits (NGCs) for Peripheral Nerve Injury Repair. Polymers (Basel), 2018. 10(7).

12. Raza, C., et al., Repair strategies for injured peripheral nerve: Review. Life Sci, 2020. 243: p. 117308.

13. Rayner, M.L.D., et al., Controlled local release of PPARgamma agonists from biomaterials to treat peripheral nerve injury. J Neural Eng, 2020. 17(4): p. 046030.

14. Rayner, M.L.D., et al., Engineered neural tissue made using clinical-grade human neural stem cells supports regeneration in a long gap peripheral nerve injury model. Acta Biomater, 2021. 135: p. 203-213.

15. Maugeri, G., et al., The role of exercise on peripheral nerve regeneration: from animal model to clinical application. Heliyon, 2021. 7(11): p. e08281.

16. Yi, S., et al., Application of stem cells in peripheral nerve regeneration. Burns Trauma, 2020. 8: p. tkaa002.

17. Yousefi, F., et al., Novel approaches using mesenchymal stem cells for curing peripheral nerve injuries. Life Sci, 2019. 221: p. 99-108.

18. Kubiak, C.A., et al., Stem-cell-based therapies to enhance peripheral nerve regeneration. Muscle Nerve, 2020. 61(4): $\mathrm{p}$. 449-459.

19. Fissel, J.A. and M.H. Farah, The influence of BACE1 on macrophage recruitment and activity in the injured peripheral nerve. J Neuroinflammation, 2021. 18(1): p. 71.

20. Gonzalez, D. and M.L. Allende, Current Advances in Comprehending Dynamics of Regenerating Axons and AxonGlia Interactions after Peripheral Nerve Injury in Zebrafish. Int J Mol Sci, 2021. 22(5).

21. Pellegatta, M. and C. Taveggia, The Complex Work of Proteases and Secretases in Wallerian Degeneration: Beyond Neuregulin-1. Front Cell Neurosci, 2019. 13: p. 93.

22. Alvites, R.D., et al., Olfactory Mucosa Mesenchymal Stem Cells and Biomaterials: A New Combination to Regenerative Therapies after Peripheral Nerve Injury, in Mesenchymal Stem Cells - Isolation, Characterization and Applications. 2017.

23. Isvoranu, G., E. Manole, and M. Neagu, Gait Analysis Using Animal Models of Peripheral Nerve and Spinal Cord Injuries. Biomedicines, 2021. 9(8).

24. Leite, A.P.S., et al., Heterologous fibrin sealant potentiates axonal regeneration after peripheral nerve injury with reduction in the number of suture points. Injury, 2019. 50(4): p. 834-847.

25. Meena, P., et al., Advances and clinical challenges for translating nerve conduit technology from bench to bed side for peripheral nerve repair. Cell Tissue Res, 2021. 383(2): p. 617-644.

26. Rao, F., et al., Aligned chitosan nanofiber hydrogel grafted with peptides mimicking bioactive brain-derived neurotrophic factor and vascular endothelial growth factor repair long-distance sciatic nerve defects in rats. Theranostics, 2020. 10(4): p. 1590-1603.

27. Lanier, S.T., et al., Evolving Techniques in Peripheral Nerve Regeneration. J Hand Surg Am, 2021. 46(8): p. 695-701.

28. Fairbairn, N.G., et al., Improving Outcomes in Immediate and Delayed Nerve Grafting of Peripheral Nerve Gaps Using Light-Activated Sealing of Neurorrhaphy Sites with Human Amnion Wraps. Plast Reconstr Surg, 2016. 137(3): p. 887895.

29. Paskal, A.M., et al., Polyethylene Glycol: The Future of Posttraumatic Nerve Repair? Systemic Review. Int J Mol Sci, 2019. 20(6).

30. Kornfeld, T., et al., Spider silk nerve graft promotes axonal regeneration on long distance nerve defect in a sheep model. Biomaterials, 2021. 271: p. 120692.

31. Ghiani, C.A., et al., Exercise decreases myelin-associated glycoprotein expression in the spinal cord and positively modulates neuronal growth. Glia, 2007. 55(9): p. 966-75.

32. Modrak, M., et al., Peripheral nerve injury and myelination: Potential therapeutic strategies. J Neurosci Res, 2020. 98(5): p. 780-795.

33. MacEwan, M.R., et al., Therapeutic electrical stimulation of injured peripheral nerve tissue using implantable thin-film wireless nerve stimulators. J Neurosurg, 2018: p. 1-10.

34. Rosso, M.P.O., et al., Photobiomodulation Therapy (PBMT) in Peripheral Nerve Regeneration: A Systematic Review. Bioengineering (Basel), 2018. 5(2).

35. Carvalho, C.R., J.M. Oliveira, and R.L. Reis, Modern Trends for Peripheral Nerve Repair and Regeneration: Beyond the Hollow Nerve Guidance Conduit. Front Bioeng Biotechnol, 2019. 7: p. 337.

36. Carvalho, C.R., et al., Engineering Silk Fibroin-Based Nerve Conduit with Neurotrophic Factors for Proximal Protection after Peripheral Nerve Injury. Adv Healthc Mater, 2021. 10(2): p. e2000753. 
37. Hellenbrand, D.J., et al., Functional recovery after peripheral nerve injury via sustained growth factor delivery from mineral-coated microparticles. Neural Regen Res, 2021. 16(5): p. 871-877.

38. Yildiran, H., M.S. Macit, and G. Ozata Uyar, New approach to peripheral nerve injury: nutritional therapy. Nutr Neurosci, 2020. 23(10): p. 744-755.

39. Bojanic, C., et al., Human umbilical cord derived mesenchymal stem cells in peripheral nerve regeneration. World J Stem Cells, 2020. 12(4): p. 288-302.

40. Caseiro, A.R., et al., Human umbilical cord blood plasma as an alternative to animal sera for mesenchymal stromal cells in vitro expansion - A multicomponent metabolomic analysis. PLoS One, 2018. 13(10): p. e0203936.

41. Saffari, S., et al., The interaction of stem cells and vascularity in peripheral nerve regeneration. Neural Regen Res, 2021. 16(8): p. 1510-1517.

42. L, P.K., et al., The mesenchymal stem cell secretome: A new paradigm towards cell-free therapeutic mode in regenerative medicine. Cytokine Growth Factor Rev, 2019. 46: p. 1-9.

43. Jahromi, M., S. Razavi, and A. Bakhtiari, The advances in nerve tissue engineering: From fabrication of nerve conduit to in vivo nerve regeneration assays. J Tissue Eng Regen Med, 2019. 13(11): p. 2077-2100.

44. Lavorato, A., et al., Mesenchymal Stem Cell Treatment Perspectives in Peripheral Nerve Regeneration: Systematic Review. Int J Mol Sci, 2021. 22(2).

45. Zhao, J., et al., Dose-effect relationship and molecular mechanism by which BMSC-derived exosomes promote peripheral nerve regeneration after crush injury. Stem Cell Res Ther, 2020. 11(1): p. 360.

46. Shi, H., et al., Bone marrow-derived neural crest precursors improve nerve defect repair partially through secreted trophic factors. Stem Cell Res Ther, 2019. 10(1): p. 397.

47. Diogo, C.C., et al., The use of sheep as a model for studying peripheral nerve regeneration following nerve injury: review of the literature. Neurol Res, 2017. 39(10): p. 926-939.

48. Rhode, S.C., J.P. Beier, and T. Ruhl, Adipose tissue stem cells in peripheral nerve regeneration-In vitro and in vivo. J Neurosci Res, 2021. 99(2): p. 545-560.

49. Sumarwoto, T., et al., Role of adipose mesenchymal stem cells and secretome in peripheral nerve regeneration. Ann Med Surg (Lond), 2021. 67: p. 102482.

50. Luo, L., et al., Application of bioactive hydrogels combined with dental pulp stem cells for the repair of large gap peripheral nerve injuries. Bioact Mater, 2021. 6(3): p. 638-654.

51. Chalisserry, E.P., et al., Therapeutic potential of dental stem cells. Journal of Tissue Engineering, 2017.8.

52. Bonaventura, G., et al., Dental mesenchymal stem cells and neuro-regeneration: a focus on spinal cord injury. Cell Tissue Res, 2020. 379(3): p. 421-428.

53. Rayner, M.L.D., et al., Quantifying regeneration in patients following peripheral nerve injury. J Plast Reconstr Aesthet Surg, 2020. 73(2): p. 201-208.

54. Torre, P. and A.I. Flores, Current Status and Future Prospects of Perinatal Stem Cells. Genes (Basel), 2020. 12(1).

55. ArefNezhad, R., H. Motedayyen, and A. Mohammadi, Therapeutic Aspects of Mesenchymal Stem Cell-Based Cell Therapy with a Focus on Human Amniotic Epithelial Cells in Multiple Sclerosis: A Mechanistic Review. Int J Stem Cells, 2021. 14(3): p. 241-251.

56. Liu, Q.W., et al., Characteristics and Therapeutic Potential of Human Amnion-Derived Stem Cells. Int J Mol Sci, 2021. 22(2).

57. Guy, R., et al., Human Muscle Progenitor Cells Overexpressing Neurotrophic Factors Improve Neuronal Regeneration in a Sciatic Nerve Injury Mouse Model. Front Neurosci, 2019. 13: p. 151.

58. Alvites, R.D., et al., Rat Olfactory Mucosa Mesenchymal Stem/Stromal Cells (OM-MSCs): A Characterization Study. International Journal of Cell Biology, 2020. 2020: p. 1-21.

59. Alvites, R.D., et al., Combined Use of Chitosan and Olfactory Mucosa Mesenchymal Stem/Stromal Cells to Promote Peripheral Nerve Regeneration In Vivo. Stem Cells Int, 2021. 2021: p. 6613029.

60. Gonzalez-Gonzalez, A., et al., Mesenchymal stem cells secretome: The cornerstone of cell-free regenerative medicine. World J Stem Cells, 2020. 12(12): p. 1529-1552.

61. Muhammad, S.A., N. Nordin, and S. Fakurazi, Regenerative potential of secretome from dental stem cells: a systematic review of preclinical studies. Rev Neurosci, 2018. 29(3): p. 321-332.

62. Raoofi, A., et al., Bone Marrow Mesenchymal Stem Cell Condition Medium Loaded on PCL Nanofibrous Scaffold Promoted Nerve Regeneration After Sciatic Nerve Transection in Male Rats. Neurotox Res, 2021. 39(5): p. 1470-1486. 
63. Yu, J., et al., Schwann-like cell conditioned medium promotes angiogenesis and nerve regeneration. Cell Tissue Bank, 2021.

64. Martins, L.F., et al., Mesenchymal stem cells secretome-induced axonal outgrowth is mediated by BDNF. Sci Rep, 2017. 7(1): p. 4153.

65. Eggers, R., et al., Lentiviral vector-mediated gradients of GDNF in the injured peripheral nerve: effects on nerve coil formation, Schwann cell maturation and myelination. PLoS One, 2013. 8(8): p. e71076.

66. Homs, J., et al., Schwann cell targeting via intrasciatic injection of AAV8 as gene therapy strategy for peripheral nerve regeneration. Gene Ther, 2011. 18(6): p. 622-30.

67. Bernstein, D.T., et al., Does the combination of erythropoietin and tapered oral corticosteroids improve recovery following iatrogenic nerve injury? Injury, 2016. 47(8): p. 1819-23.

68. Sundem, L., et al., Erythropoietin Enhanced Recovery After Traumatic Nerve Injury: Myelination and Localized Effects. J Hand Surg Am, 2016. 41(10): p. 999-1010.

69. Tseng, K.C., et al., 4-Aminopyridine promotes functional recovery and remyelination in acute peripheral nerve injury. EMBO Mol Med, 2016. 8(12): p. 1409-1420.

70. Gladman, S.J., et al., Improved outcome after peripheral nerve injury in mice with increased levels of endogenous omega3 polyunsaturated fatty acids. J Neurosci, 2012. 32(2): p. 563-71.

71. Okada, K., et al., Methylcobalamin increases Erk1/2 and Akt activities through the methylation cycle and promotes nerve regeneration in a rat sciatic nerve injury model. Exp Neurol, 2010. 222(2): p. 191-203.

72. Suzuki, K., et al., Electrospun nanofiber sheets incorporating methylcobalamin promote nerve regeneration and functional recovery in a rat sciatic nerve crush injury model. Acta Biomater, 2017. 53: p. 250-259.

73. Huff, T.C., et al., Vitamin C regulates Schwann cell myelination by promoting DNA demethylation of pro-myelinating genes. J Neurochem, 2021. 157(6): p. 1759-1773.

74. Yow, Y.Y., et al., Therapeutic Potential of Complementary and Alternative Medicines in Peripheral Nerve Regeneration: A Systematic Review. Cells, 2021. 10(9).

75. Fornasari, B.E., et al., Natural-Based Biomaterials for Peripheral Nerve Injury Repair. Front Bioeng Biotechnol, 2020. 8: p. 554257.

76. Rodriguez-Sanchez, D.N., et al., 3D-printed nerve guidance conduits multi-functionalized with canine multipotent mesenchymal stromal cells promote neuroregeneration after sciatic nerve injury in rats. Stem Cell Res Ther, 2021. 12(1): p. 303.

77. Jahromi, M., et al., Regeneration of Rat Sciatic Nerve Using PLGA Conduit Containing Rat ADSCs with Controlled Release of BDNF and Gold Nanoparticles. J Mol Neurosci, 2021. 71(4): p. 746-760.

78. Onode, E., et al., Bioabsorbable nerve conduits three-dimensionally coated with human induced pluripotent stem cellderived neural stem/progenitor cells promote peripheral nerve regeneration in rats. Sci Rep, 2021. 11(1): p. 4204.

79. Bagher, Z., et al., Hesperidin promotes peripheral nerve regeneration based on tissue engineering strategy using alginate/chitosan hydrogel: in vitro and in vivo study. International Journal of Polymeric Materials and Polymeric Biomaterials, 2020. 70(5): p. 299-308.

80. Yang, G.H., et al., 4D Bioprinting: Technological Advances in Biofabrication. Macromol Biosci, 2019. 19(5): p. e1800441.

81. Geuna, S., et al., In vitro models for peripheral nerve regeneration. Eur J Neurosci, 2016. 43(3): p. 287-96.

82. Alvites, R.D., et al., Small Ruminants and Its Use in Regenerative Medicine: Recent Works and Future Perspectives. Biology (Basel), 2021. 10(3).

83. R, D.A., et al., Establishment of a Sheep Model for Hind Limb Peripheral Nerve Injury: Common Peroneal Nerve. Int J Mol Sci, 2021. 22(3). 